\title{
The Dependencies of X-Ray Conductivity and X-Ray Luminescence of ZnSe Crystals on the Excitation Intensity
}

\author{
V. Ya. Degoda, ${ }^{1}$ M. Alizadeh $\left(\mathbb{D},{ }^{1}\right.$ N. O. Kovalenko, ${ }^{2}$ and N. Yu. Pavlova ${ }^{3}$ \\ ${ }^{1}$ Taras Shevchenko National University of Kyiv, 64 Volodymyrs'ka Street, 01601 Kyiv, Ukraine \\ ${ }^{2}$ Institute for Single Crystals NAS of Ukraine, 61001, Nauki Ave, Kharkiv, Ukraine \\ ${ }^{3}$ National Pedagogical Dragomanov University, 9 Pyrogova Street, 01601 Kyiv, Ukraine \\ Correspondence should be addressed to M. Alizadeh; trefoilsymbol@gmail.com
}

Received 28 November 2017; Accepted 25 February 2018; Published 29 May 2018

Academic Editor: Jan A. Jung

Copyright (C) 2018 V. Ya. Degoda et al. This is an open access article distributed under the Creative Commons Attribution License, which permits unrestricted use, distribution, and reproduction in any medium, provided the original work is properly cited.

\begin{abstract}
This work studies the conductivity and luminescence of ZnSe single crystals under X-ray irradiation. The experimentally derived lux-ampere characteristics of the X-ray conductivity for ZnSe crystals have a sublinear behavior within the temperature range from 8 to $420 \mathrm{~K}$. The theoretical analysis of the conductivity kinetics at X-ray excitation showed that the value of maximum accumulated lightsum at deep traps does not depend on radiation intensity. However, regarding shallow and phosphorescent traps, the strength of accumulated lightsum depends on the intensity of exciting irradiation. Specifically, these shallow traps and phosphorescent traps cause the sublinear behavior of lux-ampere characteristics in the semiconductor material.
\end{abstract}

\section{Introduction}

Zinc Selenide (ZnSe) [1-7] is a topic to be investigated. This material was well studied and well researched among the most promising wide-bandgap semiconductors (WBG or WBGS) of the II-VI semiconductor group $\left(\mathrm{A}^{\mathrm{II}} \mathrm{B}^{\mathrm{VI}}\right.$ or $\left.\mathrm{A}^{2} \mathrm{~B}^{6}\right)$. Nowadays, $\mathrm{ZnSe}$ is widely used to create shortwave semiconductor electronics and display systems $[7,8]$. Primarily, it is due to the recently learned ability to produce high-quality single crystals of a relatively large scale. As a result, another promising direction emerged. These single crystals are used as detectors of indirect ionizing radiation ( $\gamma$-radiation scintillators) [8-14] and direct conversion of the energy of high-energy radiation into the electric current (semiconductor detectors) [6,15-17]. Moreover, they are also used in the production of lenses and IR-radiation windows $[8,9,18,19]$. The application of $\mathrm{ZnSe}$ crystals as ionizing radiation detectors has become possible after developing the technology to produce high-quality crystals [20-22] with a low concentration of impurities and high resistivity $10^{10}-10^{12} \Omega \cdot \mathrm{cm}$. The single crystals have a rather large effective atomic number $\left(Z_{\text {eff }}=32\right)$ and a large band gap width $(2.7 \mathrm{eV}$ at $300 \mathrm{~K})$, which makes them a promising material to create X-ray detectors, which do not require cooling and can operate at high temperatures (up to $450 \mathrm{~K}$ ) [17]. These findings make the studies of X-ray conductivity (XRC) and X-ray luminescence (XRL) in ZnSe single crystals topical.

Lux-ampere characteristics of XRC and X-ray luminescence are one of the main features of semiconductor detectors of ionizing radiation. The dependence of conduction current on the irradiation intensity under constant electric field strength $\left(I_{\mathrm{X}}-i_{\mathrm{XRC}}\right)$ is referred to as the lux-ampere characteristic (LAC), while the dependence of luminescence intensity on the excitation intensity $\left(I_{\mathrm{X}}-J_{\mathrm{XRL}}\right)$ is referred to as the lux-luminescent characteristic (LLC). Earlier, similar studies were carried out $[6,7]$ but only at the temperature of liquid nitrogen. There is no comparison between the lux-ampere and lux-luminescent characteristics or the explanation of the obtained nonlinear dependencies.

The work aimed to experimentally investigate the spectra of luminescence and conductivity and their dependencies on the intensity of X-ray excitation for the ZnSe single crystals at various temperatures and explain the sublinear dependencies of the LAC of the X-ray conductivity and LLC of the X-ray luminescence. 


\section{Experimental Details}

In this work, we studied the conductivity and luminescence of $\mathrm{ZnSe}$ single crystals under the excitation by X-ray quanta. The specially undoped ZnSe crystals were grown from the precleaned batch, to obtain crystals with a minimum impurity concentration and a maximum resistivity $\left(\rho \geq 10^{12} \Omega . c m\right)$. The polished samples of $18 \times 9 \times 2 \mathrm{~mm}^{2}$ were prepared from different boules. It turned out that the luminescence spectra and the conductivity values did not differ much for these samples; therefore, the main complex of studies was carried out for one sample. We should note that the concentrations of free electrons in such crystals without excitation are $10^{2}$ $10^{4} \mathrm{~cm}^{-3}$.

To study the conductivity, the two three-layer metal contacts were sprayed onto one large surface of the sample by the resistive method. The chemical composition of each layer was specially selected to obtain ohmic contacts for the dark conductivity with good adhesion. Copper conductors were soldered to the contacts for the conductivity measurements. The contacts consisted of the strips of rectangular shape 5 $\mathrm{mm}$ long and $1 \mathrm{~mm}$ wide, with $5 \mathrm{~mm}$ distance between them. A stabilized voltage from 0 to $1000 \mathrm{~V}$ was applied to one electrode, while another contact was connected to a nanoamperemeter. The nanoamperemeter allowed measuring the value of the conduction current from $1 \mathrm{pA}$ to $10 \mathrm{pA}$ with an accuracy of $10 \%$; from $10 \mathrm{pA}$ to $100 \mathrm{pA}$ with an accuracy of $3 \%$; and from $100 \mathrm{pA}$ to $1 \mathrm{~mA}$ with an accuracy of $<1 \%$. For all values of the conduction current, the following condition was met: the input impedance of a nanoamperemeter is several orders of magnitude smaller than the electrical resistance of a $\mathrm{ZnSe}$ sample. The nanoamperemeter is a part of a specially developed measuring unit that allows selecting the voltage change mode: manual, stepwise, and monotonic, at which the rate of voltage increase can be changed. The study of the conduction current was carried out in a vacuum $(<1 \mathrm{~Pa})$.

It was preliminarily established by the thermal EMF method that the samples have the dark $n$-type conductivity.

The complex experimental studies of X-ray luminescence (XRL) and X-ray conductivity (XRC) were conducted. Their lux-ampere characteristics (dependencies of the conduction current $I_{\mathrm{X}}-i_{\mathrm{XRC}}$; the luminescence intensity of the different luminescence bands $I_{\mathrm{X}}-J_{630}$ and $I_{\mathrm{X}}-J_{970}$ on the intensity of Xray excitation $I_{X}$ ); the dose dependencies of phosphorescence (P) and current relaxation (CR) at the excitation temperatures of $8,85,295$, and $420 \mathrm{~K}$ were investigated. The sample was placed in the cryostat, which allowed using various temperature modes within $8-500 \mathrm{~K}$. The samples were heated using an electric furnace built into the cryostat (800 $\mathrm{W})$ and cooled by liquid nitrogen or helium.

The X-ray excitation was performed by the integral radiation of the $\mathrm{X}$-ray tube $\mathrm{BKhV7}(\mathrm{Re}, 20 \mathrm{kV}, 3 \div 25 \mathrm{~mA}$, which corresponds to $\left.I_{\mathrm{X}}=0.0762 \div 0.635 \mathrm{~mW} / \mathrm{cm}^{2}\right)$ through the beryllium window of the cryostat in the perpendicular direction to the sample surface. All X-ray irradiations were absorbed within the sample. The distance from the anode of the X-ray tube to the sample was $120 \mathrm{~mm}$, which provided for maximum intensity of the X-ray irradiation of 0.635 $\mathrm{mW} / \mathrm{cm}^{2}$. When changing the current of X-ray tube $i_{\mathrm{X} \text {-tube }}$, while the voltage is constant, the radiation intensity $I_{X}$ varies in proportion to the tube current, while the shape of the spectrum remains unchanged.

The conduction current and the luminescence radiation of the sample were recorded simultaneously. The luminescence was registered via two channels: integrally and spectrally. For those passing through one quartz window of the cryostat, the integral glow of the sample (if necessary through an optical filter) was focused by a quartz lens on a photocathode PMT-106. For those passing through another quartz window of the cryostat, the luminescence radiation was directed through a high-speed monochromator MDR2 with quartz condensers and recorded by the photoelectric multipliers: PMT-106 in the visible region or PMT-83 (in the cooling mode) in the IR region. All spectra were adjusted taking into account the spectral sensitivity of the recording system. And at the translation of the spectra from the wavelength scale $(\mathrm{nm})$ to the quanta energy scale $(\mathrm{eV})$, the spectra were corrected to the spectral radiation density.

The experimental lux-ampere dependencies of luminescence and conductivity $\left(I_{\mathrm{X}}-i_{\mathrm{XRC}}, I_{\mathrm{X}}-J_{630}\right.$ and $\left.I_{\mathrm{X}}-J_{970}\right)$ were measured in two modes: an increase $(5 \rightarrow 25 \mathrm{~mA})$ and a decrease $(25 \rightarrow 5 \mathrm{~mA})$ of X-ray intensity. Under irradiation for more than 5 minutes for each value of the excitation intensity, the divergence of the LAC curves in both modes is practically absent.

Upon terminating the excitation, both the phosphorescence (by two channels) and the current's relaxation were recorded for 10 minutes. The dose of the sample irradiation (from 0.6 to $4600 \mathrm{~mJ} / \mathrm{cm}^{2}$ ) was determined by the exposure time (from $5 \mathrm{~s}$ to $2 \mathrm{~h}$ ) and the excitation intensity (tube's current was $5 \mathrm{~mA}$ and $25 \mathrm{~mA}$ ).

The peculiarity of this work measurements was that the registration of the luminescence and conductivity of the sample were carried out simultaneously. This simultaneous registration is essential because it allows obtaining more detailed and reliable information about the processes within the sample.

\section{The Results of the Experiments}

3.1. The X-Ray Luminescence Spectra of ZnSe Single Crystals. The obtained distinctive spectra of XRL for the samples of $\mathrm{ZnSe}$ single crystals in the wavelength range from 400 to $1200 \mathrm{~nm}$ at various temperatures and excitation intensity levels are presented in Figures 1, 2, and 3. Under the high temperatures $(410 \mathrm{~K})$, due to the temperature quenching of the luminescence, the XRL intensity was so low that it was impossible to register the XRL spectra. At higher sensitivity of the registration system (by two orders of magnitude in the spectral range from 400 to $550 \mathrm{~nm}$ ) it was impossible to register neither the edge glow nor the emission of the donor-acceptor pairs (DAP) at the temperature of $8 \mathrm{~K}$, when the luminescence is the brightest within the $\mathrm{ZnSe}$ [23]. It is also essential to note that the integral luminescence intensity at low temperatures of this $\mathrm{ZnSe}$ sample (when there is no temperature quenching of the emission bands) is only several times weaker than the integral intensity of the industrial, 


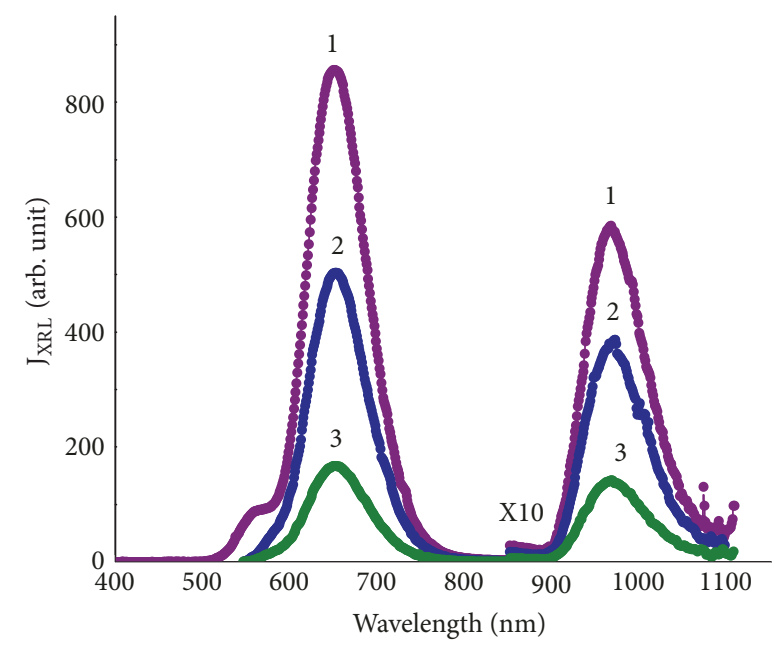

Figure 1: The distinctive XRL spectra of the ZnSe single crystal at the temperature of $8 \mathrm{~K}$ under different levels of excitation: the current of the X-ray tube, $25 \mathrm{~mA}(1) ; 15 \mathrm{~mA}$ (2); and 5mA (3) (the $\mathrm{XRL}$ intensity is multiplied by 10 for the $970 \mathrm{~nm}$ band).

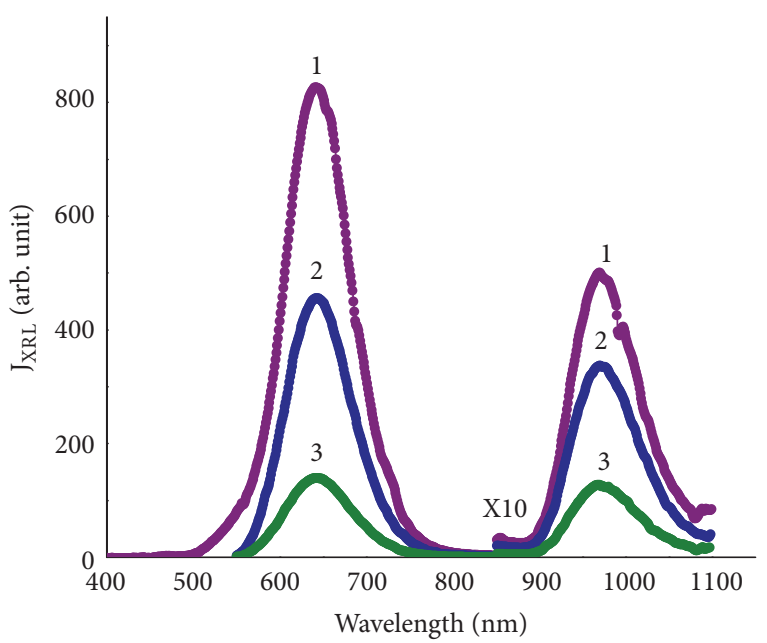

FIgure 2: The distinctive XRL spectra of the ZnSe single crystal at the temperature of $85 \mathrm{~K}$ under different levels of excitation: the current of the X-ray tube, $25 \mathrm{~mA}(1) ; 15 \mathrm{~mA}$ (2); and $5 \mathrm{~mA}$ (3) (the $\mathrm{XRL}$ intensity is multiplied by 10 for the $970 \mathrm{~nm}$ band).

the brightest X-ray luminescent $\mathrm{ZnS}$ - $\mathrm{Cu}$. It indicates a slight nonradiative loss of the excitation energy and, accordingly, high quality of these $\mathrm{ZnSe}$ crystals.

The XRL spectra of the investigated samples of $\mathrm{ZnSe}$ crystals consist of two main luminescence bands with maximums at $630 \mathrm{~nm}(1.92 \mathrm{eV})$ and $970 \mathrm{~nm}(1.28 \mathrm{eV})$. The ratio of the intensities of these bands varies for different crystals. According to papers [12, 21-23], the luminescence band with a maximum at $630 \mathrm{~nm}$ is due to the crystal complex center, which includes a $\mathrm{Zn}$ vacancy, while the luminescence band with the maximum at $970 \mathrm{~nm}$ is caused by the complex center with the Se vacancy or the Cu impurity [24, 25].

When the intensity of the X-ray excitation changes almost by an order of magnitude, the spectral positions of the

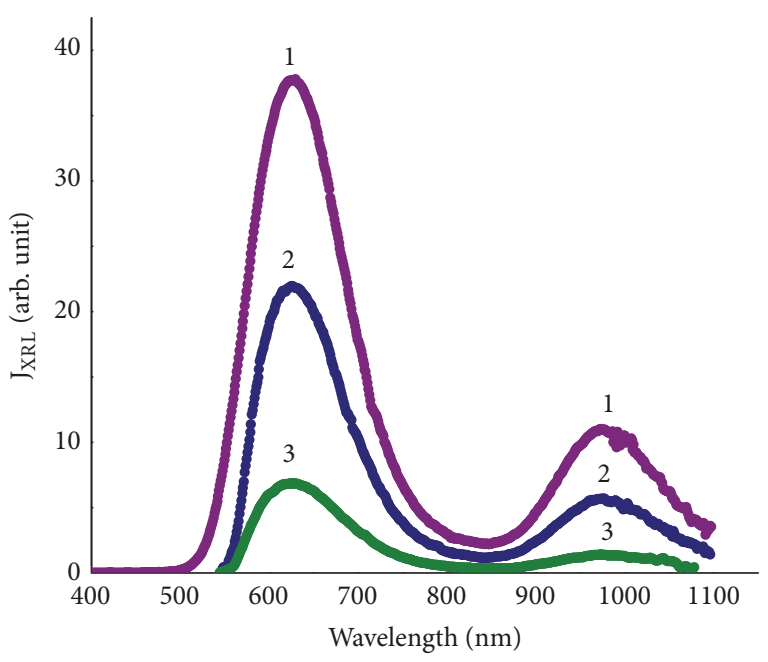

FIGURE 3: The distinctive XRL spectra of the ZnSe single crystal at the temperature of $295 \mathrm{~K}$ under different levels of excitation: the current of the X-ray tube, $25 \mathrm{~mA}(1) ; 15 \mathrm{~mA}(2)$; and $5 \mathrm{~mA}$ (3).

maximums and the bands' forms do not change. Therefore, the LLC measurements (IX-J630 and IX-J970) were carried out at the spectral peaks of the bands.

3.2. LAC of the Conductivity and LLC of the Luminescence of the ZnSe Crystals. LAC of the conductivity and LLC of the luminescence show the character of the increase in the conduction current $i_{\text {XRC }}$ and the luminescence intensity $J_{\text {XRL }}$ under the intensity increase of the X-ray or $\gamma$-radiation $I_{X}$, while the condition $I_{\mathrm{X}} \sim i_{\mathrm{X} \text {-tube }}$ is satisfied. These characteristics are essential for the scintillation and semiconductor detectors of ionizing radiation.

LAC of the conductivity $\left(I_{\mathrm{X}}-i_{\mathrm{XRC}}\right)$ and LLC of the luminescence $\left(I_{\mathrm{X}}-J_{630}\right.$ and $\left.I_{\mathrm{X}}-J_{970}\right)$ for the $\mathrm{ZnSe}$ samples were measured at different temperatures. Figure 4 shows the $I_{\mathrm{X}}-i_{\mathrm{XRC}}$ dependencies of the nonlinear behavior. Moreover, the XRC has a sublinear dependence. These dependencies were derived at feeble electric field strength $(8 \mathrm{~V} / \mathrm{cm})$ when the volt-ampere characteristics of the X-ray conductivity are still linear. Thus, such weak electric fields do not influence the luminescence and conductivity [6].

For the X-ray luminescence, the dependencies $I_{\mathrm{X}}-J_{630}$ and $I_{X}-J_{970}$, obtained at temperatures of 8,85 and $295 \mathrm{~K}$, either are linear or have a small superlinearity (Figures 5 and 6). It should be noted that after the X-ray irradiation the phosphorescence and the relaxation of the conduction current are observed in $\mathrm{ZnSe}$ samples. Therefore, all the graphs for LAC and LLC are presented after subtracting the phosphorescence and current relaxation.

Similar LLC of photoluminescence is also observed in other crystals [26-29]. The experimental dependencies $I_{\mathrm{X}}-i_{\mathrm{XRC}}, I_{\mathrm{X}}-J_{630}$, and $I_{\mathrm{X}}-J_{970}$ for the $\mathrm{ZnSe}$ samples cannot be accounted for by classical theories of the luminescence kinetics [26-28] and the conductivity [29-32]. Moreover, these theories do not take into account the above processes in correlation to each other. Therefore, the processes of 


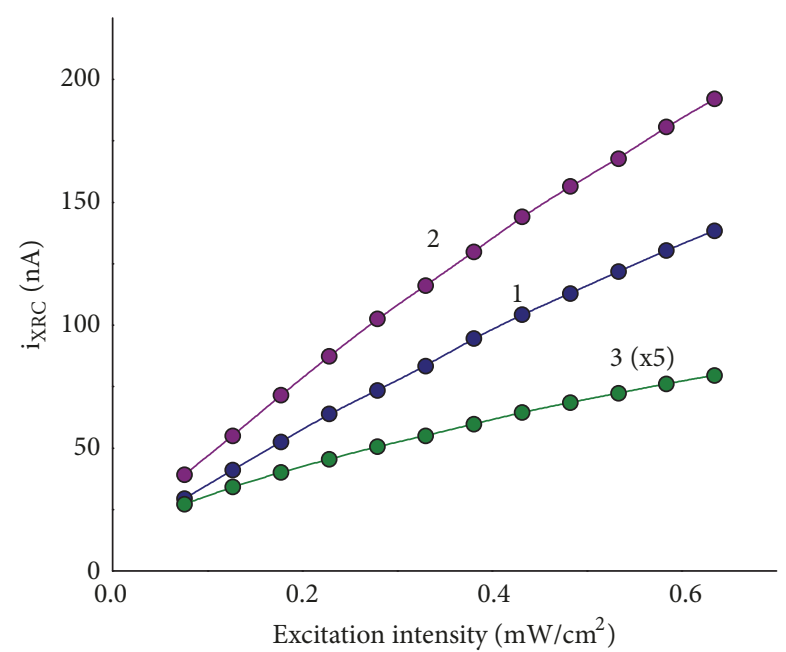

FIGURE 4: Lux-ampere characteristics of XRC of the ZnSe sample at various temperatures: $8 \mathrm{~K}(1), 85 \mathrm{~K}(2)$, and $295 \mathrm{~K}$ (3) (the intensity of the field in the sample equals $8 \mathrm{~V} / \mathrm{cm}$ ).

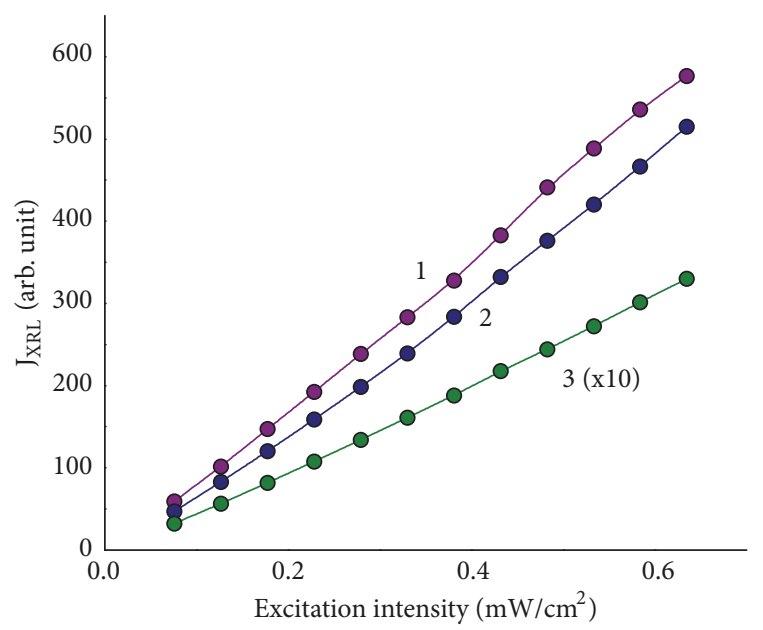

FIGURE 5: Lux-ampere characteristics of XRL of the ZnSe sample at the irradiation wavelength of $630 \mathrm{~nm}$ under various temperatures: 8 K (1), 85 K (2), and $295 \mathrm{~K}(3)$.

conductivity and recombination luminescence should be considered in correlation and explain the peculiar features of LAC and LLC, in particular for the high-resistance ZnSe crystals.

3.3. Dose Dependencies of the Phosphorescence Intensity. The accumulated lightsum in the sample during the X-ray excitation is manifested in the form of phosphorescence and current relaxation after excitation, lasting up to tens of minutes. Moreover, the main contribution to the luminescence and current makes one trap, which is called phosphorescent at the excitation temperature. While at further heating of a sample, the lightsum is manifested in the form of TSL and TSC. The accumulated lightsum is the charge carriers localized within the traps and the same number of the recharged recombination centers. It is known [33] that electrons in

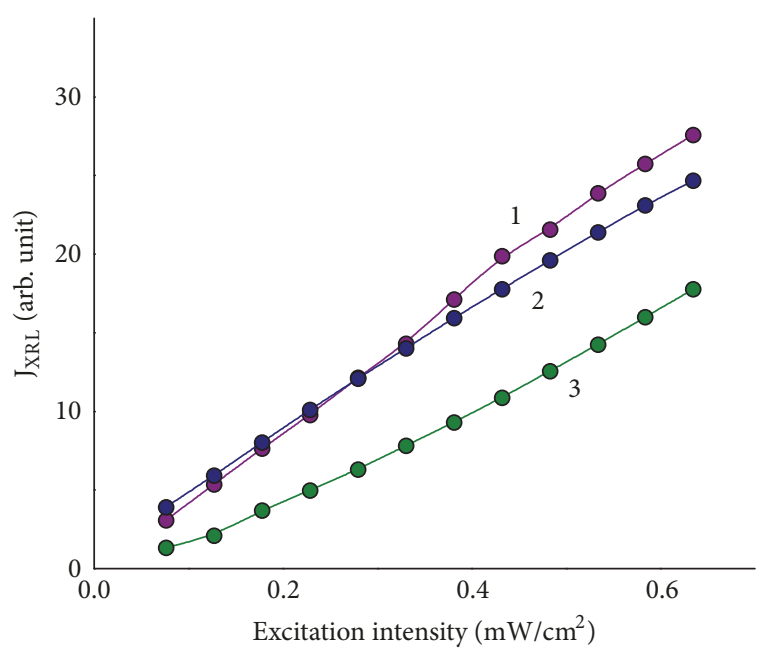

FIGURE 6: LuX-ampere characteristics of XRL of the ZnSe sample at the irradiation wavelength of $970 \mathrm{~nm}$ under the temperatures: $8 \mathrm{~K}$ (1), $85 \mathrm{~K}(2)$, and $295 \mathrm{~K}(3)$.

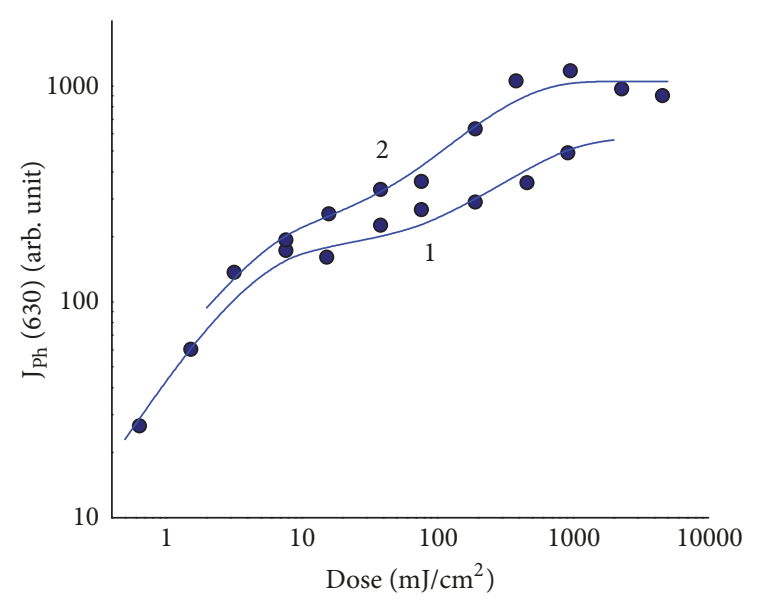

FIGURE 7: Dose dependencies of the phosphorescence intensity $J_{\mathrm{Ph}}$ of the ZnSe sample $\left(t_{\mathrm{ph}}=30 \mathrm{~s}\right)$ at registration within $630 \mathrm{~nm}$ band at the temperature of $85 \mathrm{~K}$ under different intensities of the X-ray radiation: $i_{\mathrm{X} \text {-tube }}=5 \mathrm{~mA}$ (1) and $25 \mathrm{~mA}$ (2). The solid lines show the theoretical dependency (10).

ZnSe are the free charge carriers, which determine the photoconductivity current.

The most logical is to investigate the dose dependencies of phosphorescence at $85 \mathrm{~K}$. In this case, it has a higher intensity than at 8 and $295 \mathrm{~K}$. Secondly, at this temperature the values of the general concentrations of shallow and deep traps are commensurable (have the same order). It should not be forgotten that, after each dose of irradiation and the registration of phosphorescence, the sample must be heated to $420 \mathrm{~K}$ to empty all traps.

Figures 7 and 8 show the dependencies of the phosphorescence intensity $J_{\mathrm{Ph}}\left(t_{\mathrm{ph}}=30 \mathrm{~s}\right.$ after terminating excitation $)$ on the obtained radiation dose. Using two different intensities of the X-ray radiation allows comparing the accumulated lightsums (the concentration of the recharged local centers) 


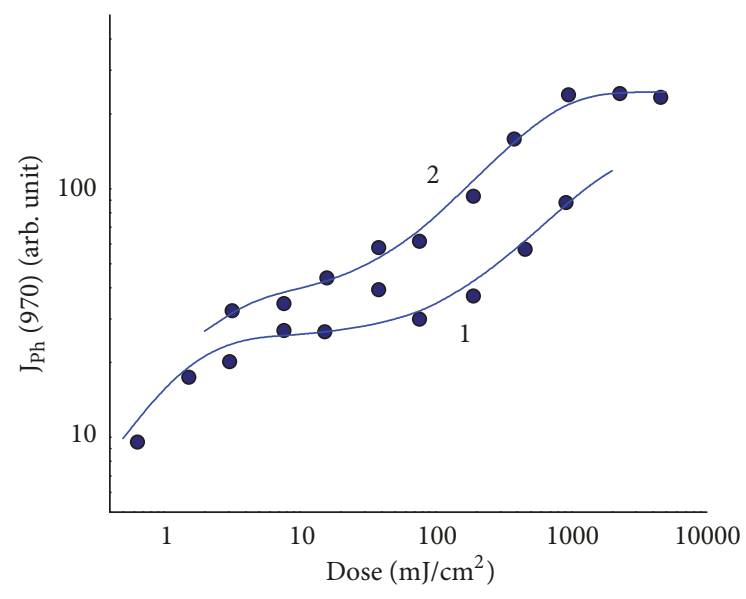

FIGURE 8: Dose dependencies of the phosphorescence intensity $J_{\mathrm{Ph}}$ of the ZnSe sample (for $30 \mathrm{~s}$ upon terminating excitation) at the registration within $970 \mathrm{~nm}$ band at the temperature of $85 \mathrm{~K}$ under different intensities of the X-ray radiation: $i_{\mathrm{X} \text {-tube }}=5 \mathrm{~mA}(1)$ and 25 $\mathrm{mA}$ (2). The solid lines show the theoretical dependency (10).

at equal doses of irradiation but obtained for a different period. In Figures 7 and 8 the circles show the experimental data, while solid lines show the theoretical dependency (10).

According to the experimental data, in all cases, the concentration of the recharged centers (even when reaching the saturation level) will be large at the more increased intensity of the $\mathrm{X}$-ray radiation.

\section{Analysis of the Experimental Results}

As the semiconductor absorbs X-ray radiation, the free electrons and holes are generated, the amount of which is proportional to the energy of the absorbed X-ray quanta. Therefore, the amount of the produced (in the semiconductor) free electrons and holes $G$ per unit of time is proportional to the absorbed energy of the X-ray or $\gamma$-radiation $[34,35]$.

The sublinear behavior of LAC of the X-ray conductivity $\left(I_{\mathrm{X}}-i_{\mathrm{XRC}}\right)$ in $\mathrm{ZnSe}$ crystals indicates that when the intensity of $\mathrm{X}$-ray excitation $I_{\mathrm{X}}$ increases, the concentration of free charge carriers, which determine the conduction current $i_{\mathrm{XRC}}$ also increases sublinearly. The concentrations of free electrons and holes are uniquely related to the concentration of generated carriers $G$ via an average lifetime of free electrons $\left(\tau^{-}\right)$and holes $\left(\tau^{+}\right)$:

$$
\begin{aligned}
N^{-} & =\tau^{-} G \\
\text { and } P^{+} & =\tau^{+} G .
\end{aligned}
$$

Therefore, the sublinear behavior of $I_{\mathrm{X}}-i_{\mathrm{XRC}}$ indicates a monotonous decrease of the charge carriers' lifetime by those signs that causes the conduction process. According to [32], these carriers are electrons in the $\mathrm{ZnSe}$ crystals. The lifetime of free electrons in the conduction band is determined by the concentration of different types of traps $v_{\mathrm{i}}$, their filling $n_{\mathrm{i}}$, and concentration of the recharged recombination centers $p_{j}$ (i.e., the filled traps for holes):

$$
\tau^{-}=\frac{1}{u^{-}\left(\sum_{i} \sigma_{i}^{-}\left(v_{i}-n_{i}\right)+\sigma_{j}^{-} p_{j}\right)},
$$

where $u^{-}, u^{+}$are thermal velocities of free electrons and holes, respectively; $\sigma_{\mathrm{i}}{ }^{-}$is the capture cross section of free electrons at the traps of $i$-type; $\sigma_{j}{ }^{+}$is the capture cross section of the free holes at recombination centers; and $\sigma_{j}{ }^{-}$is the capture cross section of free electrons at the recharged luminescence center (the recombination cross section). Usually, for high-quality crystalline phosphors, the concentrations of luminescence centers are much higher than the traps concentrations $\left(v_{\mathrm{j}}>>\right.$ $\left.\Sigma v_{\mathrm{i}}\right)$. At the point of initial radiation, the lifetime of free electrons is determined as follows:

$$
\tau^{-}=\frac{1}{u^{-} \sum_{i} \sigma_{i}^{-} v_{i}} .
$$

Assuming that the probability of recombination of the free holes with localized electrons can be neglected, in comparison with the probability of their localization at the luminescence centers, the lifetime of free holes can be written as follows:

$$
\begin{aligned}
\tau^{+} & =\frac{1}{u^{+} \sigma_{j}^{+}\left(v_{j}-p_{i}\right)} \\
\text { and } \tau_{0}^{+} & =\frac{1}{u^{+} \sigma_{j}^{+} v_{j}} .
\end{aligned}
$$

At $v_{\mathrm{j}} \gg \Sigma v_{\mathrm{i}}$, the lifetime of free holes is much shorter than that of free electrons. During X-ray irradiation, the lightsum is accumulated; i.e., the concentrations of localized electrons at traps $n_{\mathrm{i}}$ and the concentrations of the recharged luminescence centers $p_{\mathrm{j}}$ will increase. However, the law of charge conservation is required to satisfy the balance equation:

$$
\sum_{i} n_{i}=p_{j}
$$

The irradiation of the semiconductor causes the increased lifetime of free holes $\tau^{+}$in comparison with the reference value $\tau_{0}{ }^{+}$. But the execution of the inequality $p_{j}<<v_{j}$ allows ignoring the change of $\tau^{+}$during the irradiation. For the free electrons, the denominator in relation (2) can be rewritten as follows:

$$
\begin{aligned}
& u^{-}\left(\sum_{i} \sigma_{i}^{-}\left(v_{i}-n_{i}\right)+\sigma_{j}^{-} p_{j}\right) \\
& \quad=u^{-}\left[\sum_{i} \sigma_{i}^{-} v_{i}+\left(\sigma_{j}^{-} p_{j}-\sum_{i} \sigma_{i}^{-} n_{i}\right)\right] .
\end{aligned}
$$

If the capture cross section of the recombination $\sigma_{j}{ }^{-}$and localization $\sigma_{\mathrm{i}}{ }^{-}$for free electrons would be the same, then, by the balance equation (5), the expression in parentheses (6) should be equal to zero and value of $\tau^{-}$would not change during the irradiation process. But since $\sigma_{\mathrm{j}}{ }^{-} / \sigma_{\mathrm{i}}{ }^{-}>1$ (due to the extra electric charge of the recharged recombination 
center), the difference $\left(\sigma_{\mathrm{j}}^{-} p_{\mathrm{j}}-\Sigma \sigma_{\mathrm{i}}{ }^{-} v_{\mathrm{i}}\right)>0$. It means that the lifetime of free electrons will decrease as the accumulated lightsum increases, in accordance with the kinetic theory of luminescence and conductivity, for three types of traps (shallow (i-1)-type, phosphorescent i-type, and deep (i+1)type), as well as one luminescence center [36]. The value of the maximum accumulated lightsum on deep traps for electrons and holes does not depend on the intensity of excitation, if there is no thermal or optical delocalization in the process of excitation. The nonradiative recombination of the electronhole pairs at nonradiative recombination centers occurs according to the same laws as in the luminescence centers. For a multicenter crystal-phosphorus model [36], when several recombination centers are considered, one of these centers can be considered as nonradiative. It fundamentally does not affect the kinetics of luminescence and conductivity, but it is necessary to establish a recombination mechanism (electron or hole) to be realized at this center. Secondly, in a multicenter crystal-phosphorus model, the nonradiative recombination of free holes in deep traps filled with electrons is taken into account.

The processes of phosphorescence and relaxation of conduction current are observed in ZnSe crystal at temperatures 8 and $85 \mathrm{~K}$. At room temperature, the relaxation of conduction current is observed, and the phosphorescence process is not registered due to the temperature quenching of for both luminescence bands at $T>100 \mathrm{~K}$. According to [36], the maximum value for the concentration of electrons $\left(n_{\mathrm{i} \infty}\right)$ localized at traps with i-type:

$$
n_{i \infty}=\sqrt{\frac{1}{4}\left[\frac{G}{w_{i}}\left(1+\frac{v_{i}}{v_{p}}\right)\right]^{2}+\frac{G v_{i}}{w_{i}}}-\frac{G}{2 w_{i}}\left(1+\frac{v_{i}}{v_{p}}\right) .
$$

The carried-out experimental verification (Figures 7 and 8) confirmed the above assumption, since the maximum value of phosphorescence intensities (at registration at both luminescence bands of 630 and $970 \mathrm{~nm}$ ) is more significant at a higher intensity of excitation. Also, [36] shows the dependence for the kinetics of lightsum accumulation at $k$ trap depending on the irradiation dose:

$$
\begin{aligned}
& n_{k}(t)=n_{k \infty} \\
& \quad \cdot \frac{1-\exp \left[-\left(n_{k \infty} / v_{k}+G / w_{k} n_{k \infty}\right) w_{k} t\right]}{1-w_{k} n_{k \infty}^{2} / G v_{k} \cdot \exp \left[-\left(n_{k \infty} / v_{k}+G / w_{k} n_{k \infty}\right) w_{k} t\right]} .
\end{aligned}
$$

This ratio for shallow traps of (i-1)-type and phosphorescent trap of i-type at the condition $w_{(\mathrm{i}-1)} v_{(\mathrm{i}-1)}, w_{\mathrm{i}} v_{\mathrm{i}}>\mathrm{G}$ has the following form:

$$
\begin{aligned}
n_{(i-1)}(t) & =n_{(i-1) \infty}\left[1-\exp \left(-\frac{t}{\tau_{(i-1)}}\right)\right] \\
\text { and } n_{i}(t) & =n_{i \infty}\left[1-\exp \left(-\frac{t}{\tau_{i}}\right)\right] .
\end{aligned}
$$

The accumulation of charge carries at shallow traps, and their devastation occurs faster than at deeper traps.

At the phosphorescence, the charges carriers are delocalized from all traps (shallow, phosphorescent, and deep), but



FIGURE 9: Normalized dependencies of the ratio of the intensity of the XRL $630 \mathrm{~nm}$ band to the X-ray conductivity current on the excitation intensity at temperatures: $8 \mathrm{~K}(1), 85 \mathrm{~K}(2)$, and $295 \mathrm{~K}(3)$.

the registered intensity of the phosphorescence $J_{\mathrm{Ph}}$ will be determined, primarily, by the additive sum of the recombinations of delocalized electrons from small and phosphorescent traps:

$$
\begin{gathered}
J_{\mathrm{Ph}} \sim n_{(i-1) \infty} w_{(i-1)}\left[1-\exp \left(-\frac{t}{\tau_{(i-1)}}\right)\right] \\
+n_{i \infty} w_{i}\left[1-\exp \left(-\frac{t}{\tau_{i}}\right)\right],
\end{gathered}
$$

where $J_{\mathrm{Ph}}$ is the phosphorescence intensity at any point of time after excitation and time $t_{\mathrm{X}}$ in (10) is the duration of $\mathrm{X}$ ray irradiation. The ratio (10) was used for the approximation of experimental dose dependencies of the phosphorescence (Figures 7 and 8 ).

According to the experimental results (Figures 7 and 8), at a lower intensity of irradiation, the accumulated lightsum in the crystal will be smaller, even at similar doses of radiation. This is true for both types of luminescence centers, which cause broad electronic-vibrational luminescence bands with peaks at 630 and $970 \mathrm{~nm}$.

Another evidence of the reduction of maximum accumulated lightsum at the decreased intensity of excitation during long-term irradiation can be the dependence of the ratio of luminescence intensity to the conduction current value on the intensity of excitation intensity. Since the intensity of luminescence $J_{\mathrm{XRL}}$ is proportional to the production of the free charge carriers' concentration and the concentration of the recharged luminescence centers $p_{\mathrm{j}}$, while the conduction current value $i_{\text {XRC }}$ is proportional to the free charge carriers' concentration only, their ratio can be written as follows:

$$
\frac{J_{\mathrm{XRL}}}{i_{\mathrm{XRC}}} \sim p_{\mathrm{j}}\left(I_{\mathrm{X}}\right) .
$$

Figures 9 and 10 show the dependencies of the ratio of luminescence intensity for 630 and $970 \mathrm{~nm}$ bands to the X-ray 




FIGURE 10: Normalized dependencies of the ratio of the intensity of the XRL $970 \mathrm{~nm}$ band to the X-ray conductivity current on the excitation intensity at temperatures: $8 \mathrm{~K}$ (1), $85 \mathrm{~K}$ (2), and $295 \mathrm{~K}$ (3).

conduction current on excitation intensity at temperatures: 8, 85, and $295 \mathrm{~K}$ for respective luminescence centers. For calculation purposes, the experimental dependencies are shown in Figures 4-6.

In both cases of XRL registration, the increase in the concentration of recharged luminescence centers occurs when the intensity of X-ray excitation increases. It means that when the intensity of the X-ray excitation increases, according to (2), the free electron lifetime in conduction band decreases. As a result, the concentration of free electrons increases unproportionally and sublinearly for the excitation intensity. As a consequence, we derive the sublinear $I_{\mathrm{X}}-i_{\mathrm{XRC}}$ behavior in the semiconductor.

The influence of accumulated lightsum within the crystal on the $I_{\mathrm{X}}-i_{\mathrm{XRC}}$ behavior indicates that, to obtain a correct experimental dependence, it is necessary to get the maximum accumulated lightsum in the sample for each excitation intensity. Otherwise, different values of current for one excitation intensity can be obtained; i.e., we will observe "hysteresis" at the increase or decrease of the X-ray excitation intensity $[24,25]$.

The classical theory of photoconductivity [26-32] was developed for a simple crystalline phosphor model (one type of trap and one type of recombination center). In case of an extended period of stationary excitation for the concentration of free charge carriers, it provides for the dependence $N^{-} \sim$ $\sqrt{I_{X}}$ and a similar dependence for the concentration of the recharged recombination centers $p_{j} \sim \sqrt{I_{X}}$. It ensures a proportional dependence for the luminescence intensity and for the current $-\sqrt{I_{X}}$. If the experimental dependencies $I_{\mathrm{X}}-i_{\mathrm{XRC}}$ are approximated by the provided function, we derive the exponent $\sim 0.8 \div 0.9$, which significantly differ from 0.5 .

Therefore, the conduction current dependence on the intensity of the $\mathrm{X}$-ray or $\gamma$-radiation can vary in the range from linear $i_{\mathrm{XRC}} \sim I_{\mathrm{X}}$ to $i_{\mathrm{XRC}} \sim \sqrt{I_{X}}$. The degree of the sublinearity depends on the traps' concentrations in the sample. The higher the values of the phosphorescence intensities and relaxation current in the semiconductor are, the more significant the nonlinearity of the lux-ampere characteristic is.

The results of the X-ray and UV excitation of luminescence and conductivity of ZnSe crystals [37] pave the way to study zinc selenide as semiconductor detectors of ionizing radiation for the detection of ionizing radiation.

\section{Conclusions}

The sublinear behavior of the dependency of the conduction current curves on the intensity of X-ray or $\gamma$-radiation $\left(I_{\mathrm{X}}-i_{\mathrm{XRC}}\right)$ can be explained by the presence of several types of traps for free charge carriers and recombination centers in the semiconductor. According to the theoretical analysis of X-ray conductivity kinetics, the maximum accumulated lightsum at deep traps does not depend on the excitation intensity. Also, for shallow and phosphorescent traps, the accumulated lightsum depends on the excitation intensity: it increases with increasing excitation intensity. According to experimental data analysis, we can assume that the higher the concentration of such defects is, i.e., the higher the intensity of phosphorescence and current relaxation is, the closer the lux-ampere characteristics will be to $i_{\mathrm{XRC}} \sim \sqrt{I_{X}}$. While the concentration of defects (i.e., traps for free charge carriers) decrease, the lux-ampere characteristics will approximate to the linear ones.

\section{Conflicts of Interest}

The authors declare that they have no conflicts of interest.

\section{References}

[1] A. N. Georgobiani and M. K. Sheinkman, Physics of AIIBVI Compounds, Nauka, Moscow, Russia, 1986.

[2] D. D. Nedeoglo and A. V. Simashkevich, Electric and Luminescence Properties of Zinc Selenide, Shtiintsa, Kishinev, Moldova, 1984.

[3] V. I. Gavrilenko, A. M. Grekhov, D. V. Korbutyak, and V. G. Litovchenko, Optical Properties of Semiconductors, Nauk. Dumka, Kiev, Ukraine, 1987.

[4] N. K. Morozova, V. A. Kuznetsov, and V. D. Ryzhikov, Zinc Selenide: Receiving and optical properties, Science, Moscow, Russia, 1992.

[5] G. A. Bordovsky, "X-ray conductivity of High Ohmic Semiconductors," Sorosovsky Educational Magazine, vol. 7, no. 3, pp. 8489, 2001 (Russian).

[6] V. Y. Degoda and A. O. Sofienko, "Specific features of the luminescence and conductivity of zinc selenide on exposure to X-ray and optical excitation," Semiconductors, vol. 44, no. 5, pp. $1-7,2010$.

[7] V. Y. Degoda and G. P. Podust, "X-ray conductivity of ZnSe single crystals," Semiconductors, vol. 50, no. 5, pp. 579-585, 2016.

[8] V. S. Vavilov, "Physics and applications of wide band gap semiconductors and their practical applications," Physics-Uspekhi, no. 3, pp. 287-296, 1994 (Russian).

[9] L. V. Atroshchenko, S. F. Burachas, L. P. Galchinetski, B. V. Grinev, V. D. Ryzhikov, and N. G. Starzhinskii, Scintillation 
Crystals and Ionization Radiation Detectors on Their Base, Nauk. Dumka, Kiev, Ukraine, 1998.

[10] B. V. Grinyov, V. D. Ryzhikov, and V. P Semynozhenko, Scintillation Detectors and Radiation Control Systems on Their Basis, Naukova Dumka, Kiev, Ukraine, 2007.

[11] D. B. Elmurotova and E. M. Ibragimova, "Strengthening electroluminescence of $\mathrm{ZnSe}$ single crystals (Te, O) after $\gamma$-radiation," The Physic and The Technology of Semiconductors, vol. 41, no. 10, 2007.

[12] A. Nasr, A. Aboshosha, and M. Ashour, "Performance Evaluation of Phototransistors and their Behavior under Gamma Radiation Effects," in Proceedings of the EG0800227 The Second All African IRPA Regional Radiation Protection Congress, Ismailia, Egypt, 2007.

[13] I. Dafinei, M. Fasoli, F. Ferroni et al., "Low temperature scintillation in ZnSe crystals," IEEE Transactions on Nuclear Science, vol. 57, no. 3, pp. 1470-1474, 2010.

[14] N. Starzhinskiy, B. Grinyov, I. Zenya, V. Ryzhikov, L. Gal'chinetskii, and V. Silin, "New trends in the development of AIIBVI-based scintillators," IEEE Transactions on Nuclear Science, vol. 55, no. 3, pp. 1542-1546, 2008.

[15] V. D. Ryzhikov et al., "Properties of semiconductor scintillators $\mathrm{ZnSe}(\mathrm{Te}, \mathrm{O})$ and integrated scintielectronic radiation detectors based thereon," IEEE Transactions on Nuclear Science, vol. 48, no. 1, pp. 356-359, 2001.

[16] Y. H. Cho, S. H. Park, W. G. Lee et al., "Comparative study of a CsI and a $\mathrm{ZnSe}(\mathrm{Te} / \mathrm{O})$ scintillation detector's properties for a gamma-ray measurement," Journal of Nuclear Science and Technology, vol. 45, no. 5, pp. 534-537, 2014.

[17] V. Ryzhikov, G. Tamulaitis, N. Starzhinskiy, L. Gal'chinetskii, A. Novickovas, and K. Kazlauskas, "Luminescence dynamics in ZnSeTe scintillators," Journal of Luminescence, vol. 101, no. 1-2, pp. 45-53, 2003.

[18] A. O. Sofiienko and V. Y. Degoda, "X-ray induced conductivity of $\mathrm{ZnSe}$ sensors at high temperatures," Radiation Measurements, vol. 47, no. 1, p. 27, 2012.

[19] A. O. Sofiienko, V. Ya. Degoda, and V. N. Kilin, "Basic Model of the Stationary X-ray Induced Conductivity of Wide-Gap Semiconductors," Global Journal of Science Frontier Research Physics \& Space Science, vol. 12, no. 3, 2012.

[20] M. S. Brodyn, V. Y. Degoda, A. O. Sofiienko, B. V. Kozhushko, and V. T. Vesna, "Monocrystalline ZnSe as an ionising radiation detector operated over a wide temperature range," Radiation Measurements, vol. 65, pp. 36-44, 2014.

[21] E. Krause, H. Hartmann, J. Menninger et al., "Influence of growth non-stoichiometry on optical properties of doped and non-doped ZnSe grown by chemical vapour deposition," Journal of Crystal Growth, vol. 138, pp. 75-80, 1994.

[22] V. M. Koshkin, A. Ya. Dulfan, V. D. Ryzhikov, L. P. Gal'chinetskii, and N. G. Starzhinskiyet, "Thermodynamics of isovalent tellurium substitution for selenium in $\mathrm{ZnSe}$ semiconductors," Journal of Functional Materials, vol. 8, no. 4, pp. 708713, 2001.

[23] R. N. Bhargava, R. J. Seymour, B. J. Fitzpatrick, and S. P. Herko, "Donor-acceptor pair bands in ZnSe," Physical Review B, vol. 20, no. 6, pp. 2407-2419, 1979.

[24] V. Ryzhikov, B. Grinyov, S. Galkin, N. Starzhinskiy, and I. Rybalka, "Growing technology and luminescent characteristics of ZnSe doped crystals," Journal of Crystal Growth, vol. 364, pp. 111-117, 2013.
[25] N. K. Morozova, I. A. Karetnikov, V. V. Blinov, and E. M. Gavrishchuk, "A study of luminescence centers related to copper and oxygen in ZnSe," Semiconductors, vol. 35, no. 1, pp. 24-32, 2001.

[26] N. K. Morozova, I. A. Karetnikov, V. V. Blinov, and E. M. Gavrishchuk, "Studies of the infrared luminescence of $\mathrm{ZnSe}$ doped with copper and oxygen," Semiconductors, vol. 35, no. 5, pp. 512-515, 2001.

[27] J. Ji, A. M. Colosimo, W. Anwand et al., "ZnO Luminescence and scintillation studied via photoexcitation, X-ray excitation, and gamma-induced positron spectroscopy," Scientific Reports, vol. 6, Article ID 31238, 2016.

[28] A. Janotti and C. G. Van de Walle, "Fundamentals of zinc oxide as a semiconductor," Reports on Progress in Physics, vol. 72, no. 12, Article ID 126501, 2009.

[29] C. R. Varney, M. A. Khamehchi, J. Ji, and F. A. Selim, "X-ray luminescence based spectrometer for investigation of scintillation properties," Review of Scientific Instruments, vol. 83, no. 10, Article ID 103112, 2012.

[30] F. A. Selim, M. H. Weber, D. Solodovnikov, and K. G. Lynn, "Nature of native defects in ZnO," Physical Review Letters, vol. 99, no. 8, Article ID 085502, 2007.

[31] E. I. Adirovich, "Some Problems in the Theory of Luminescence of Crystals," Tech. Rep., State publishing house of technical and theoretical literature, Moscow, Russia, 1956.

[32] M. V. Fock, Introduction to Kinetics of Luminescence of Phosphor Crystals, Nauka, Moscow, Russia, 1964.

[33] V. E. Lashkarev, A. V. Lyubchenko, and M. K. Sheinkman, Nonequilibrium Processes in Photoconductors, Nauk. Dumka, Kiev, Ukraine, 1981.

[34] V. V. Antonov-Romanovski, Photoluminescence Kinetics of Phosphor Crystals, Nauka, Moscow, Russia, 1966.

[35] R. H. Bube, Photoconductivity of Solids, John Wiley \& Sons, NY, USA, 1960.

[36] V. Y. Degoda, A. F. Gumenyuk, and Y. A. Marazuev, The Kinetic of Recombination Luminescence and Conductivity of Crystalophosphors, Taras Shevchenko National University of Kyiv VPT, Kiev, Ukraine, 2016.

[37] V. Y. Degoda, M. Alizadeh, N. O. Kovalenko, and N. Y. Pavlova, "V-I characteristics of X-ray conductivity and UV photoconductivity of ZnSe crystals," Journal of Applied Physics, vol. 123, no. 7, Article ID 075702, 2018. 

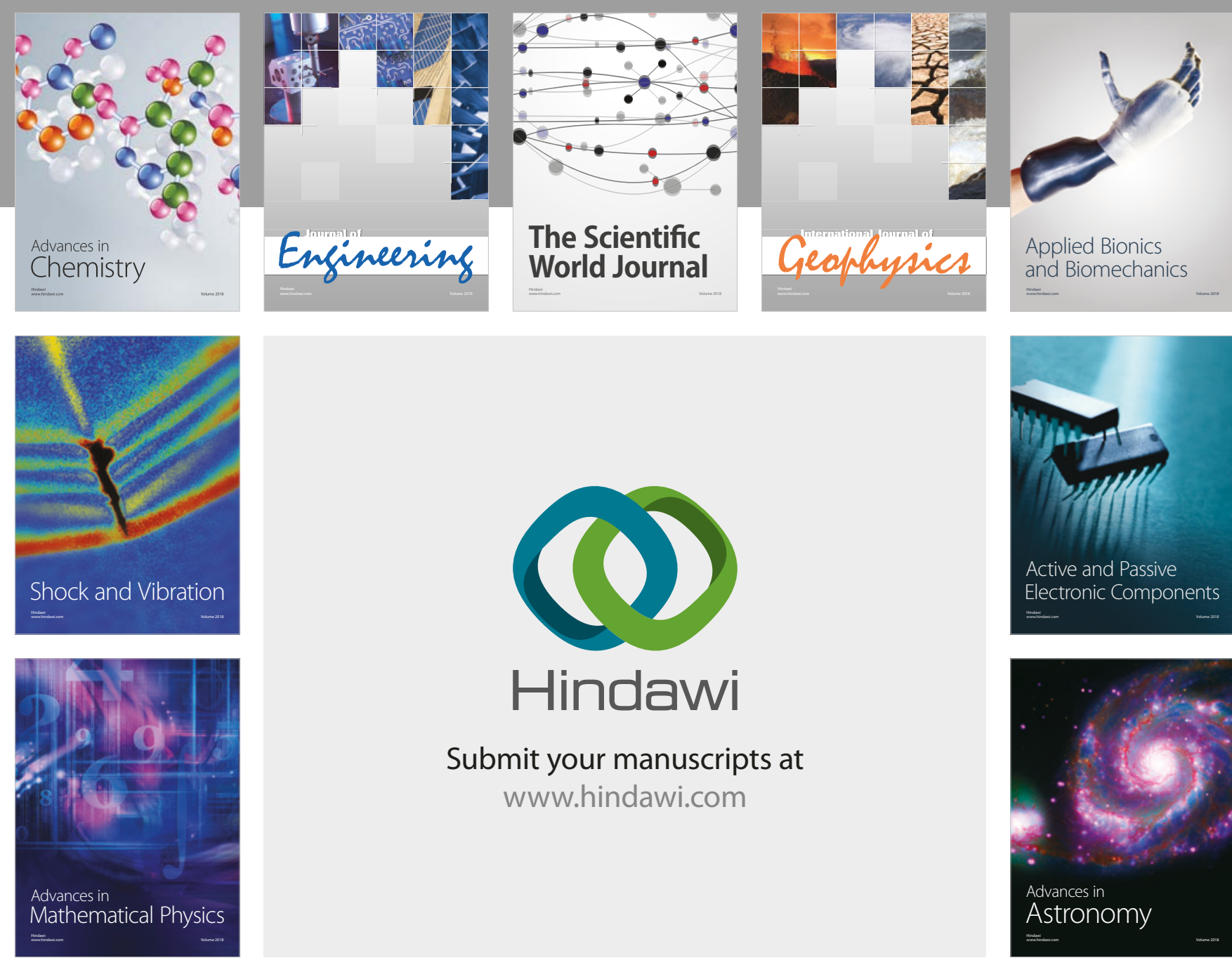

Submit your manuscripts at

www.hindawi.com

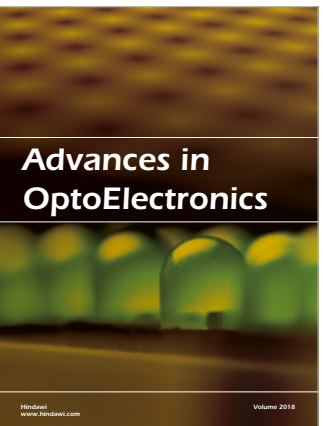

\section{Rotcting Machinery}
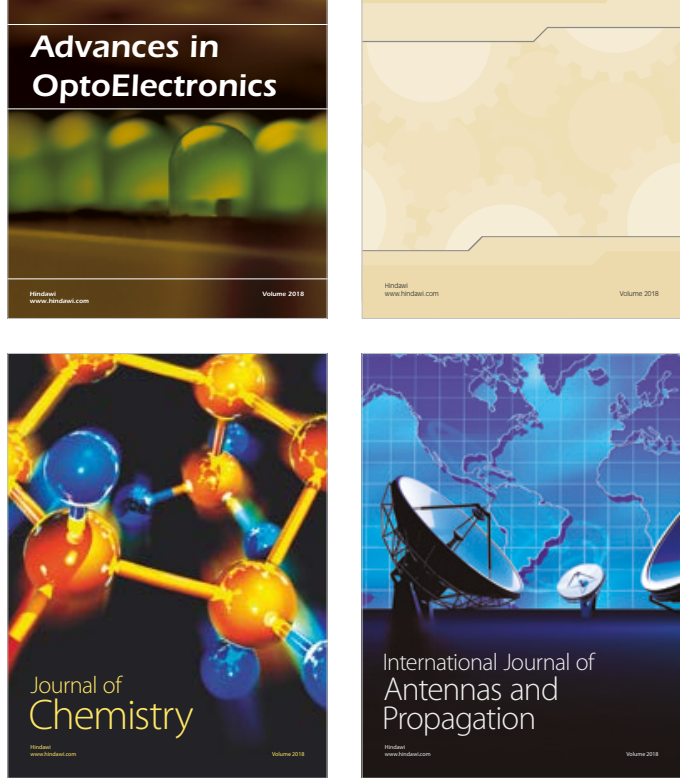

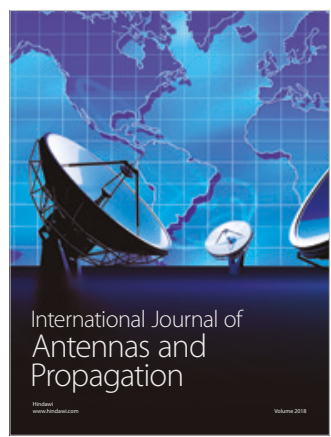

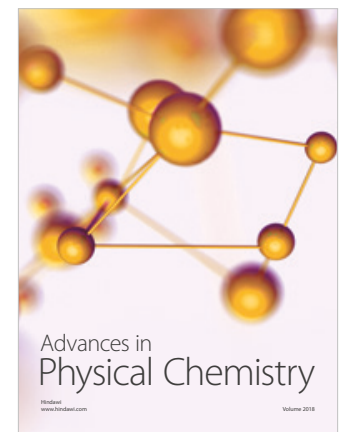

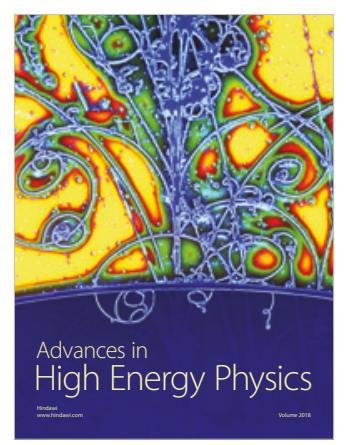

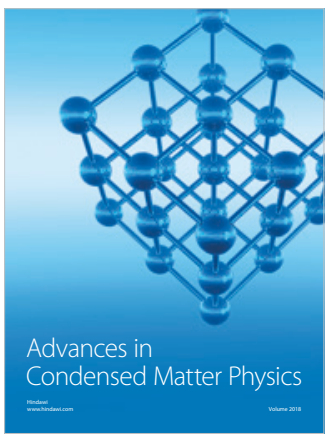

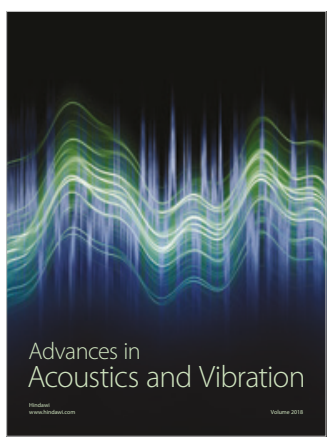

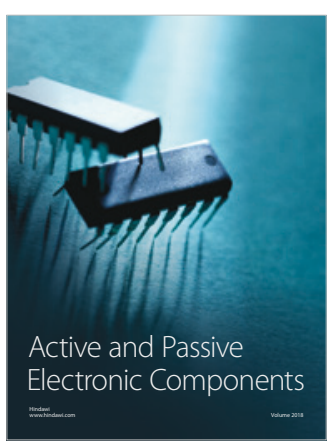
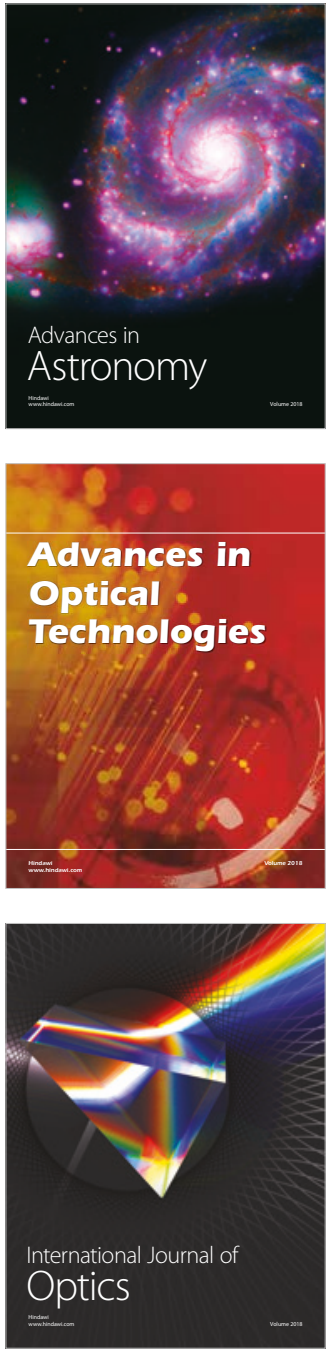